\title{
Characterisation and impact of reported and unreported exacerbations: results from ATTAIN
}

\author{
Paul W. Jones ${ }^{1}$, Rosa Lamarca², Ferran Chuecos², Dave Singh ${ }^{3}$, Alvar Agustíi, \\ Eric D. Bateman ${ }^{6}$, Gonzalo de Miquel $^{2}$, Cynthia Caracta ${ }^{7}$ and Esther Garcia Gil ${ }^{2}$
}

Affiliations: 'Division of Clinical Science, St George's, University of London, London, UK. ${ }^{2}$ R\&D Centre, Almirall, Barcelona, Spain. ${ }^{3}$ Medicines Evaluation Unit, University Hospital of South Manchester, Manchester, UK. ${ }^{4}$ Thorax Institute, Hospital Clinic, IDIBAPS, University of Barcelona, Barcelona, Spain. ${ }^{5} \mathrm{FISIB}$, Centro de Investigación Biomédica en Red Enfermedades Respiratorias (CIBERES), Mallorca, Spain. ${ }^{6}$ Dept of Medicine, University of Cape Town, Cape Town, South Africa. ${ }^{7}$ Forest Research Institute, Forest Laboratories Inc., Jersey City, NJ, USA.

Correspondence: Paul W. Jones, St George's, University of London, Cranmer Terrace, London, SW17 ORE, UK. E-mail: pjonesdsgul.ac.uk

ABSTRACT The frequency and impact of exacerbations identified using healthcare resource utilisation (HCRU) or the EXAcerbations of Chronic pulmonary disease Tool (EXACT) were compared prospectively in a 24-week, phase III trial (ATTAIN). Patients with moderate-to-severe chronic obstructive pulmonary disease received twice-daily aclidinium $200 \mu \mathrm{g}$, aclidinium $400 \mu \mathrm{g}$ or placebo. All HCRU events were reported to physicians. "EXACT-identified" events were categorised as "EXACT-reported" (detected by EXACT and reported to the physician) and "EXACT-unreported" (detected but not reported). Health status was measured using the St George's Respiratory Questionnaire (SGRQ).

Annualised EXACT-identified event rates were higher in all study arms (placebo 1.39, aclidinium $200 \mu \mathrm{g}$ 1.00 and aclidinium $400 \mu \mathrm{g} 0.98$ per patient per year) versus HCRU (placebo 0.60, aclidinium $200 \mu \mathrm{g} 0.43$ and aclidinium $400 \mu \mathrm{g} 0.40$ per patient per year). Concordance between methods was low (kappa 0.16 ). Aclidinium reduced EXACT-identified events (rate ratio versus placebo: aclidinium $200 \mu \mathrm{g} 0.72$ and aclidinium $400 \mu \mathrm{g} 0.71$; both $\mathrm{p}<0.05$ ); HCRU events were similarly reduced.

At week 24, SGRQ scores improved (-6.6 versus baseline) in patients with no event during weeks 1-12; improvements were significantly smaller in patients with HCRU events $(-3.4 ; p=0.036)$ or EXACTunreported events $(-3.0 ; \mathrm{p}=0.002)$.

Unreported events were more frequent than reported events. Both had similar negative impact on health status. Aclidinium reduced the frequency of both types of event.

@ERSpublications

There are twice as many unreported exacerbation events as reported events; both have similar impact on health status http://ow.ly/AwJVf

This article has supplementary material available from erj.ersjournals.com

Received: Feb 262014 | Accepted after revision: Aug 112014 | First published online: Sept 182014

Clinical trial: This study is registered at ClinicalTrials.gov with identifier number NCT01001494.

Support statement: This study was funded by Almirall S.A., Barcelona, Spain, and Forest Laboratories, Inc., New York, NY, USA. The study sponsors were responsible for the conception and design of the study, collection of the data and for data analysis. The sponsors placed no restrictions on statements made in the final version of the manuscript or on the decision to submit the manuscript for publication. All authors had full access to the data and take full responsibility for the content of the manuscript, including the data and analysis.

Conflict of interest: Disclosures can be found alongside the online version of this article at erj.ersjournals.com

Copyright @ERS 2014 


\section{Introduction}

Patients with chronic obstructive pulmonary disease (COPD) may experience exacerbations, defined as an acute worsening of respiratory symptoms, beyond the normal day-to-day variation, that leads to a change in medication [1]. Exacerbations of COPD (ECOPDs) worsen health status, accelerate the rate of lungfunction decline, and are associated with increased mortality risk and considerable economic cost [2-4]. Consequently, prevention of ECOPDs is an important goal in the management of patients with COPD.

In clinical studies, ECOPDs are generally assessed based on healthcare resource utilisation (HCRU), with the degree of therapeutic intervention used to define severity [5-7]. This approach has a number of limitations, including regional differences in management practices and healthcare access, and differences in the factors that influence individuals to change treatment themselves or seek medical advice [8]. Furthermore, it does not capture ECOPDs experienced by the patient but not reported to the clinician and therefore not treated [9-11]. To address this, the EXAcerbations of Chronic pulmonary disease Tool (EXACT) was developed as a standardised patient-reported outcome instrument that evaluates the frequency, severity and duration of exacerbation events, based on changes in symptoms reported directly by the patient [8].

A recent evaluation of the performance of the EXACT in three prospective randomised controlled trials of exacerbation prevention has provided evidence of the validity of the EXACT in this context [12]; however, the treatments studied did not alter exacerbation rates and the impact of unreported exacerbations was not investigated [12]. Here, we have used data from the 24-week, randomised, placebo-controlled Aclidinium To Treat Airway obstruction IN COPD patients (ATTAIN) study [13] to examine reported exacerbation events and those that were not reported but were captured by the EXACT. The aims were to: 1) compare the incidence, characteristics and degree of concordance of exacerbation events identified using HCRU criteria or the EXACT; 2) assess the effect of maintenance bronchodilator treatment with aclidinium on both types of event; and 3) investigate the impact of reported (HCRU) and unreported (identified only by the EXACT) events on health status and trough forced expiratory volume in $1 \mathrm{~s}$ (FEV1) in these patients.

\section{Methods}

Study design

ATTAIN (ClinicalTrials.gov identifier: NCT01001494) was a 24-week, double-blind, randomised, placebocontrolled, parallel-group phase III study in which patients were randomised (1:1:1) to receive aclidinium $200 \mu \mathrm{g}$, aclidinium $400 \mu \mathrm{g}$ or placebo twice daily [13]. Concomitant treatment with other long-acting bronchodilators was not allowed. Permitted medication included inhaled corticosteroids and systemic corticosteroids ( $\leqslant 10 \mathrm{mg}$ per day), if their use was stable $\geqslant 4$ weeks prior to screening, and salbutamol (as needed).

The study was conducted in accordance with the Declaration of Helsinki, International Conference on Harmonisation/Good Clinical Practice Guidelines and local regulations. The protocol was approved by independent ethics committees at each centre before study initiation. All patients gave written informed consent.

\section{Study subjects}

Male and female patients ( $\geqslant 40$ years old) with a diagnosis of stable moderate-to-severe COPD (postbronchodilator $\mathrm{FEV} 1 \geqslant 30 \%$ and $<80 \%$ of the predicted value and $\mathrm{FEV} 1 /$ forced vital capacity ratio $<70 \%$ ) were eligible [1]. Patients with an ECOPD within 6 weeks (or 3 months if hospitalisation was required) before screening or during the run-in period were excluded. The inclusion/exclusion criteria have been reported previously; prior exacerbation history was not an inclusion criterion [13].

\section{Assessment of exacerbation events}

An intent-to-treat (ITT) analysis was used for all comparisons between groups (see the online supplementary material for additional detail).

EXACT events

Patients recorded symptom severity using the EXACT patient diary, a 14-item daily diary completed at night using an electronic personal digital assistant (PDA) $[8,14]$. Information was transferred from the PDA for centralised reading each week via a modem. The recall period was "today" and patients selected the answer that best described their experience for that day. EXACT scores ranged from 0 to 100; higher scores indicated more severe symptoms $[14,15]$. An EXACT-identified event was defined as a persistent increase from baseline in total EXACT score of $\geqslant 9$ points for $\geqslant 3$ days or $\geqslant 12$ points for $\geqslant 2$ days $[14,16]$. To allow for possible baseline shifts after recovery from an event, if no further event occurred within 28 days the baseline was reset using data from post-event days 22-28 [16]. Using the EXACT, recovery is defined as an improvement of $\geqslant 9$ points from the maximum value during the 14-day period following onset of an 
event, sustained for 7 days using a 3-day rolling average, but we did not investigate recovery times in this analysis. EXACT scores and events were analysed after study completion and were not available to the investigator assessing HCRU events.

\section{HCRU events}

HCRU events were identified by the investigator at each study visit based on the investigator's assessment of a patient's symptoms (using information provided by the patient that may have included information from the electronic diary), concomitant medication use recorded using a paper diary and information on hospitalisation recorded in the case report form. HCRU events were defined as an increase in COPD symptoms for $\geqslant 2$ days that required a change in regular COPD medication and categorised as mild (selfmanaged by increasing usual COPD medication (short-acting bronchodilator and/or inhaled corticosteroids)), moderate (treated with antibiotics and/or systemic corticosteroids or an increase in systemic corticosteroids) or severe (requiring hospitalisation) [5]. Following an HCRU event, a repeat event was defined as new if the patient had stopped the additional treatment with oral steroids and antibiotics $\geqslant 14$ days previously.

Exacerbation events analysed

An HCRU event was classified as a reported (and treated) exacerbation. EXACT events were categorised as: 1) EXACT-identified (all EXACT events, both HCRU reported and unreported); 2) EXACT-reported (also identified by HCRU); and 3) EXACT-unreported (EXACT events not identified by HCRU).

\section{End-points}

Pre-specified end-points included: 1) annualised rates of HCRU events and EXACT-identified events; 2) the percentages of patients with $\geqslant 1$ HCRU event (any severity) or $\geqslant 1$ EXACT-identified event; and 3) time to first event, assessed by both the EXACT and HCRU.

A previous study suggested that following an exacerbation, health status, even in patients with no subsequent exacerbation, may take $>12$ weeks to fully recover [17]. Therefore, to examine the mediumterm impact of exacerbation events and minimise the effect of any recent exacerbations, post hoc analyses assessed changes from baseline in St George's Respiratory Questionnaire (SGRQ) total score and trough FEV1 at week 24 in patients who had either an HCRU event, an EXACT-unreported event or no exacerbation event during weeks $1-12$.

\section{Statistical analysis}

Results are presented as mean $\pm \mathrm{SD}$, least square mean $\pm \mathrm{SE}$, percentage, rate ratio $(95 \% \mathrm{CI}$; annualised exacerbation rates) or odds ratio (95\% CI; binary outcomes) as appropriate.

Poisson regression with correction for over-dispersion, logistic regression, kappa index, Kaplan-Meier estimates, Cox proportional hazards model and ANCOVA were used as appropriate (additional detail on the statistical analyses is provided in the online supplementary material). Analyses were performed using SAS v9.3 (SAS Institute Inc., Cary, NC, USA). p-values $<0.05$ were considered statistically significant.

\section{Results}

\section{Patient characteristics}

The ITT population comprised 819 patients. Overall patient characteristics have been reported previously [13]. The mean \pm SD age was $62.4 \pm 8.0$ years; most patients $(68.1 \%)$ had moderate airflow limitation and $34.7 \%$ of patients had $\geqslant 1$ HCRU event (any severity) in the year before enrolment [13].

When baseline characteristics of patients with exacerbations were compared, predicted FEV1 was lower in patients with $\geqslant 1$ HCRU event (49.1\% (95\% CI 46.8-51.4\%); $\mathrm{n}=138$ ) versus those with $\geqslant 1$ EXACTidentified event (52.2\% (95\% CI 50.6-53.9\%); $\mathrm{n}=261 ; \mathrm{p}=0.026)$ (online supplementary table S1). A similar difference was observed in patients who only had HCRU events versus patients who had EXACT-unreported events (48.2\% (95\% CI 45.0-51.5\%) versus 53.1\% (95\% CI 51.2-54.9\%); $\mathrm{p}=0.010)$. Other differences were small. Across countries, there was clear variation in HCRU event reporting compared with reporting of EXACT-identified events (fig. 1).

\section{Incidence of EXACT-identified and HCRU events}

In placebo-treated patients, annualised rates of EXACT-identified events and HCRU events were 1.39 and 0.60 per patient per year, respectively (fig. 2a). EXACT also identified a higher proportion of patients experiencing $\geqslant 1$ event versus HCRU (fig. $2 \mathrm{~b}$ ). This result was consistent for both aclidinium treatment arms (fig. $2 \mathrm{a}$ and $\mathrm{b}$ ). 


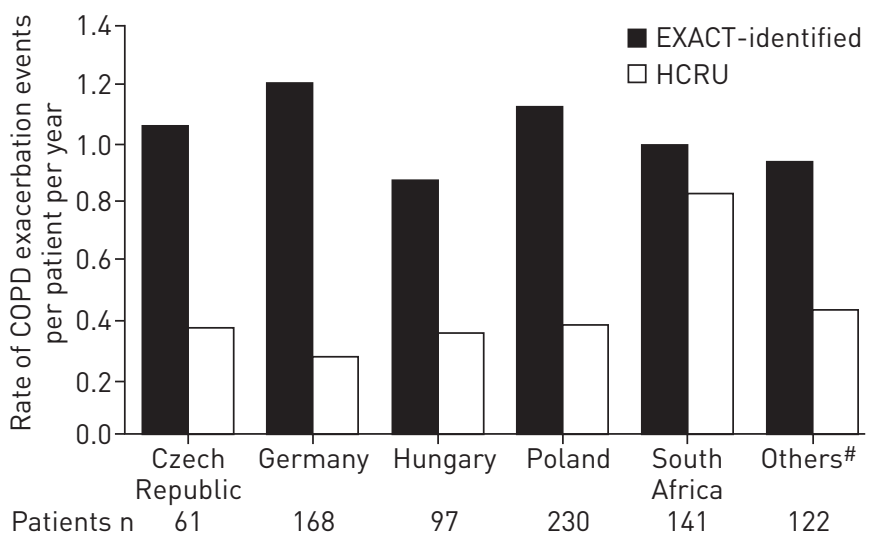

FIGURE 1 Annualised healthcare resource utilisation (HCRU) and EXAcerbations of Chronic pulmonary disease Tool (EXACT)-identified event rates by country. All treatment arms combined; intent-to-treat population. COPD: chronic obstructive pulmonary disease. "\#: France, Italy, Russia, Spain, Ukraine.

\section{Concordance between EXACT-identified and HCRU events}

Agreement between patients with EXACT-identified events and HCRU events was poor (table 1 and online supplementary fig. S2). This was due to a number of factors: 1) EXACT identified twice as many patients experiencing an event compared with HCRU $(31.9 \%(n=261 / 819)$ versus $16.8 \%(n=138 / 819)$, respectively); 2) $73.6 \%(n=192 / 261)$ of patients who had an EXACT-identified event did not have an HCRU event; 3) $12.4 \%(n=69 / 558)$ of patients who did not have an EXACT-identified event had an HCRU event; and 4) $50 \%(n=69 / 138)$ of patients who experienced an HCRU event did not have an EXACT-identified event. As a result, the kappa index of concordance between the two methods was low (0.16). Of patients who met the criteria for an HCRU event, 21.0\% $(n=29 / 138)$ required increased use of bronchodilators (mild), $71.7 \%$ $(n=99 / 138)$ were treated with antibiotics and/or corticosteroids (moderate) and $10.9 \%(n=15 / 138)$ required hospitalisation (severe). The proportion of patients who had an HCRU event but not an EXACT event was similar across all severities of HCRU events (50.5-53.3\%).

When the actual number of exacerbation events was assessed, there were 155 HCRU events $(19.4 \%$ mild, 70.3\% moderate and $10.3 \%$ severe) (online supplementary table S2) and 374 EXACT-identified events. In total, only $34.8 \%(n=54 / 155)$ of all HCRU events were also identified by the EXACT. The proportion of HCRU events identified with the EXACT was very similar across all degrees of HCRU event severity (mild $33.3 \%$, moderate $35.8 \%$ and severe $31.3 \%$ ) (online supplementary table S2).

\section{EXACT scores in EXACT events and HCRU events}

Mean EXACT scores from day -14 before an event to 28 days post-event were assessed for EXACT-reported, EXACT-unreported and HCRU-only events (online supplementary fig. S1). Patients with EXACTunreported events had significantly lower scores at baseline (day - 14 to -7 pre-event) and at event onset (day 0 ) than patients with EXACT-reported events (fig. 3 and table 2). However, absolute \pm SD differences from baseline versus day 0 were similar in both groups $(-12.3 \pm 7.3$ for EXACT-unreported events and $-12.4 \pm 8.1$

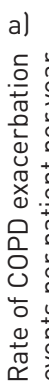

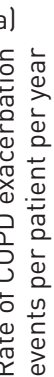

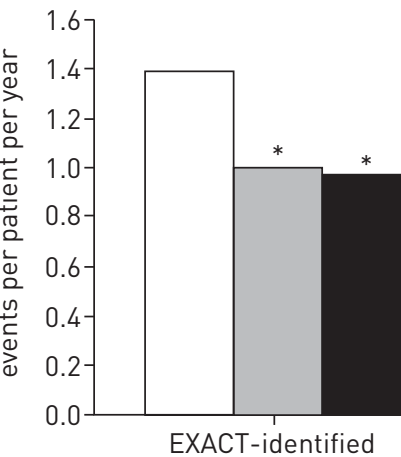

$\square$ Placebo (n=273)

$\square$ Aclidinium $200 \mu \mathrm{g}(\mathrm{n}=277)$

Aclidinium $400 \mu \mathrm{g}(\mathrm{n}=269)$

FIGURE 2 Annualised EXAcerbations of Chronic pulmonary disease Tool (EXACT)-identified and healthcare resource utilisation (HCRU) a) event rates and b) proportions of patients experiencing $\geqslant 1$ EXACT-identified event or HCRU event. Aclidinium was taken twice daily; intent-to-treat population. COPD: chronic obstructive pulmonary disease. ${ }^{*}: \mathrm{p}<0.05$ versus placebo.

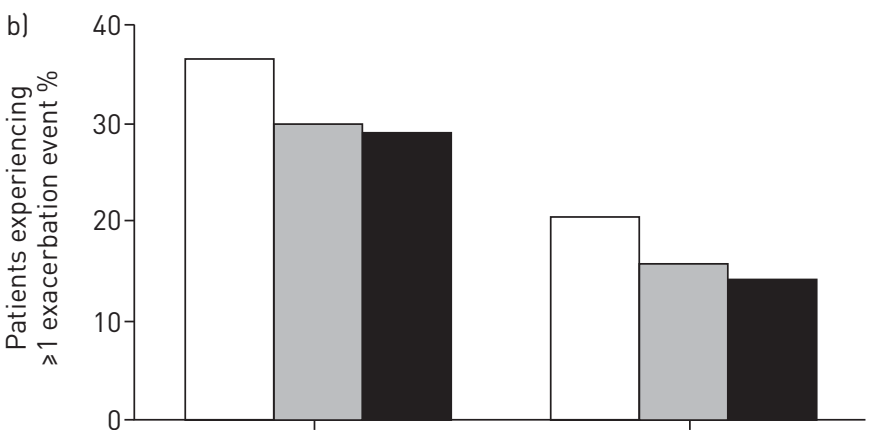

EXACT-identified
HCRU 
TABLE 1 Comparison of patients who reported $\geqslant 1$ EXACT-identified event and $\geqslant 1$ HCRU event

\begin{tabular}{|c|c|c|c|c|c|c|}
\hline & \multirow[t]{2}{*}{ Patients n } & \multicolumn{2}{|c|}{ All HCRU events } & \multicolumn{3}{|c|}{ HCRU event severity\# } \\
\hline & & $\geqslant 1$ event & No event & Mild" & Moderate $^{+}$ & Severe ${ }^{\S}$ \\
\hline Patients n & & 138 & 681 & 29 & 99 & 15 \\
\hline \multicolumn{7}{|l|}{ All EXACT events } \\
\hline$\geqslant 1$ event & 261 & $69(50.0)$ & $192(28.2)$ & 14 (48.3) & 49 (49.5) & $7(46.7)$ \\
\hline No event & 558 & $69(50.0)$ & $489(71.8)$ & $15(51.7)$ & 50 (50.5) & 8 (53.3) \\
\hline Kappa index & & \multicolumn{2}{|c|}{0.16} & NA & NA & NA \\
\hline
\end{tabular}

Data are from the intent-to-treat population and are presented as $\mathrm{n}(\%)$, unless otherwise stated. EXACT: EXAcerbations of Chronic pulmonary disease Tool; HCRU: healthcare resource utilisation; NA: not applicable. \#: patients who experienced multiple HCRU events of different severities were counted in each HCRU severity group; ": self-managed by increasing usual chronic obstructive pulmonary disease medication (short-acting bronchodilator and/or inhaled corticosteroids); ${ }^{+}$: treated with antibiotics and/or systemic corticosteroids or an increase in systemic corticosteroids; ${ }^{\S}$ : requiring hospitalisation.

for EXACT-reported events). During recovery, mean scores following EXACT-unreported events remained elevated at days $21-28$ post-event compared with the baseline period $(1.3 \pm 7.7 ; \mathrm{p}=0.007)$, whereas scores in EXACT-reported events returned to baseline $(-0.3 \pm 7.6 ; p=0.791)$ (fig. 3 and table 2$)$.

69 patients $(8.4 \%)$ experienced HCRU-only events. In these patients, EXACT scores were similar at baseline to those in patients who experienced EXACT-unreported events, increased at day 0 by only a small amount and returned to baseline during recovery (fig. 3, table 2 and online supplementary fig. S1).

\section{Effects of aclidinium on EXACT-identified and HCRU events}

Kaplan-Meier curves for time to first EXACT-identified event and time to first HCRU event (any severity) are shown in figure $4 \mathrm{a}$ and b, respectively. A Cox proportional hazards model demonstrated that time to first EXACT-identified event was significantly prolonged with both aclidinium doses versus placebo ( $\mathrm{p}=0.031$ and $\mathrm{p}=0.015$ for twice-daily aclidinium $200 \mu \mathrm{g}$ and $400 \mu \mathrm{g}$, respectively) and time to first HCRU event was significantly prolonged with twice-daily aclidinium $400 \mu \mathrm{g}$ versus placebo $(\mathrm{p}=0.040)$.

As previously reported [13], both aclidinium doses significantly reduced the annualised HCRU event rate versus placebo (aclidinium $200 \mu \mathrm{g}$ : rate ratio 0.72 (95\% CI 0.52-0.99), $\mathrm{p}=0.043$; aclidinium $400 \mu \mathrm{g}$ : rate ratio 0.67 (95\% CI $0.48-0.94), \mathrm{p}=0.020$ ) (fig. $2 \mathrm{a}$ and online supplementary material). Similar results were observed for all EXACT-identified events, with the annualised rate reduced versus placebo by $28 \%$ (rate ratio $0.72(95 \%$ CI $0.55-0.94) ; \mathrm{p}=0.017)$ with aclidinium $200 \mu \mathrm{g}$ and by $29 \%$ (rate ratio $0.71(95 \% \mathrm{CI}$ 0.54-0.93); $\mathrm{p}=0.012$ ) with aclidinium $400 \mu \mathrm{g}$ (fig. 2a and online supplementary material). The number needed to treat (NNT) to prevent one exacerbation event per patient per year was lower for EXACT (2.6 and 2.5 with aclidinium $200 \mu \mathrm{g}$ and $400 \mu \mathrm{g}$, respectively) than for HCRU (5.9 and 5.1 with aclidinium $200 \mu \mathrm{g}$ and $400 \mu \mathrm{g}$, respectively).

Aclidinium $200 \mu \mathrm{g}$ and $400 \mu \mathrm{g}$ also reduced the proportion of patients who experienced $\geqslant 1$ HCRU event (fig. 2b), but the reduction with either dose was not significantly different versus placebo. Similarly, both aclidinium doses reduced the proportion of patients experiencing $\geqslant 1$ EXACT-identified event, but the reductions were not significantly different versus placebo (fig. 2b).

The magnitude of changes in EXACT score with onset and recovery from an EXACT-identified event and an HCRU event were very similar in placebo- and aclidinium-treated patients (fig. 5).

Impact of EXACT events and HCRU events on health status and bronchodilation

To assess the medium-term effects of exacerbations on health status and bronchodilation, we assessed changes from baseline in SGRQ total score and trough FEV1 at week 24 in patients who had an exacerbation during weeks 1-12. An initial analysis was performed using all treatment arms combined (placebo, aclidinium $200 \mu \mathrm{g}$ and aclidinium $400 \mu \mathrm{g}$ ). In patients who had no exacerbations, SGRQ score at week 24 improved from baseline by -6.6 units; in patients with EXACT-unreported events this improvement was significantly smaller ( -3.0 units; $\mathrm{p}=0.002$ versus no exacerbation) (table 3 ). In patients with HCRU events, the improvement was also significantly smaller compared with patients who had no exacerbation $(-3.4$ units; $p=0.036)$ (table 3). Significantly fewer patients in the EXACT-unreported group achieved improvements in SGRQ total score greater than the minimum clinically important difference of 4 units 


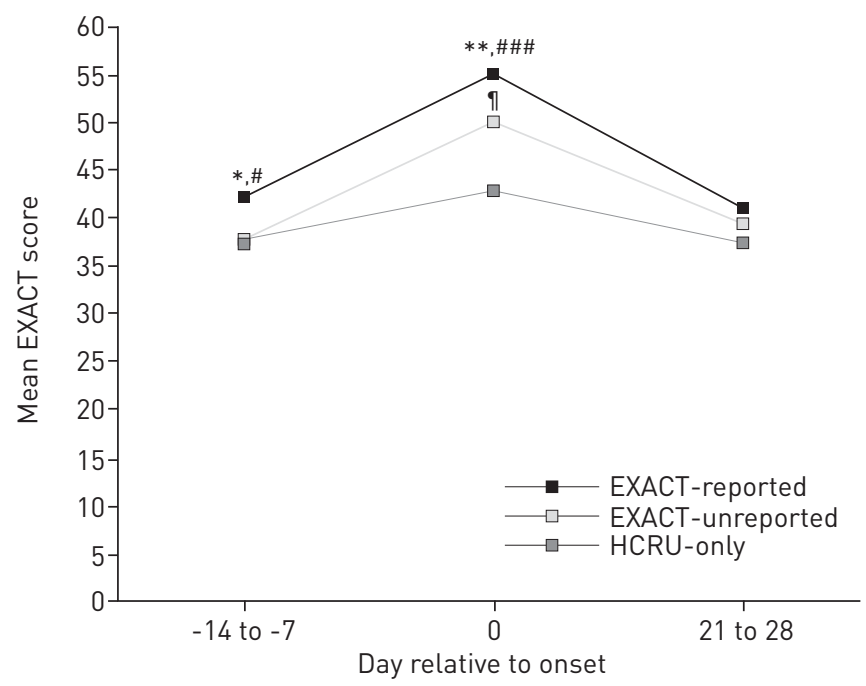

FIGURE 3 Mean EXAcerbations of Chronic pulmonary disease Tool (EXACT) scores on days -14 to -7 , day 0 and days 21 to 28 relative to onset (day 0) of an EXACT-reported event, an EXACT-unreported event and a healthcare resource utilisation (HCRU)-only event. Intent-to-treat population. Higher scores indicate more severe symptoms. ${ }^{*}: \mathrm{p}<0.05$ EXACT-reported versus EXACT-unreported events; ${ }^{* *}: \mathrm{p}<0.01$ EXACT-reported versus EXACT-unreported events; ${ }^{\#}: \mathrm{p}<0.05$ EXACT-reported versus HCRU only; ${ }^{\# \# \# ; ~} \mathrm{p}<0.001$ EXACT-reported versus HCRU only; ${ }^{\circ}: \mathrm{p}<0.001$ EXACTunreported versus HCRU only. See table 2 for more detailed data.

(SGRQ responders) compared with patients who had no exacerbations (table 3). A similar picture was seen in patients with HCRU events, but these differences were not statistically significant. Changes from baseline in trough FEV1 were not significantly different at week 24 between patients who had no exacerbations or those with EXACT-unreported or HCRU events during weeks 1-12 (table 3).

In patients who did not have an exacerbation event during weeks 1-12, both doses of aclidinium improved the SGRQ total score by $>4.5$ units at week 24 versus placebo (table 4 ). In patients with an EXACTunreported or an HCRU event, the improvements in SGRQ score were smaller and not significantly different compared with placebo (table 4). Treatment differences between both aclidinium doses are shown in online supplementary table S3.

\section{Discussion}

This is the first efficacy study to use the EXACT to compare unreported (and therefore untreated) exacerbation events with reported (and therefore treated) HCRU exacerbation events and to examine the

\section{TABLE 2 Mean EXACT scores and differences in scores for EXACT-reported, EXACT-unreported and HCRU-only events}

Days -14 to -7

\section{EXACT-reported events}

Events $\mathrm{n}$

EXACT score

EXACT-unreported events

Events $\mathrm{n}$

EXACT score

HCRU-only events

Events $\mathrm{n}$

EXACT score

\section{Change in EXACT score}

EXACT-reported versus EXACT-unreported

EXACT-unreported versus HCRU-only

EXACT-reported versus HCRU-only
Day 0

49

$42.3 \pm 10.3$

283

$55.1 \pm 12.2$

322

$37.7 \pm 12.5$

92

$37.8 \pm 11.4$

$4.6 \pm 12.2^{*}$

$0.0 \pm 12.2$

$4.6 \pm 11.0^{*}$

Days 21 to 28

Data are from the intent-to-treat population and are presented as mean \pm SD, unless otherwise stated. The events counted were those with associated e-diary records during the given period. Day 0 was the onset of an event. EXACT: EXAcerbations of Chronic pulmonary disease Tool; HCRU: healthcare resource utilisation. ${ }^{*}: \mathrm{p}<0.05$; $^{* *}: \mathrm{p}<0.01 ;{ }^{* * *}: \mathrm{p}<0.001$. 


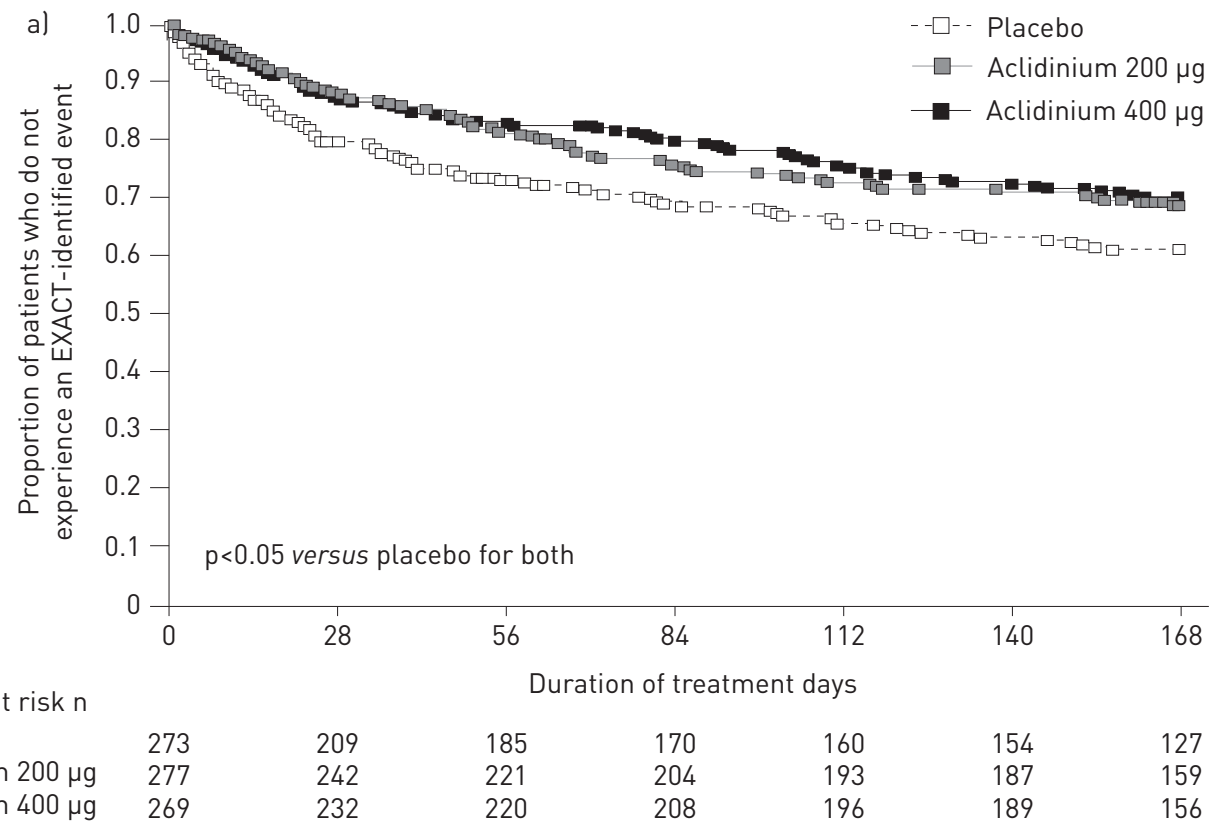

\begin{tabular}{|c|c|c|c|c|c|c|c|}
\hline Patients at risk n & \multicolumn{7}{|c|}{ Duration of treatment days } \\
\hline Placebo & 273 & 209 & 185 & 170 & 160 & 154 & 127 \\
\hline Aclidinium $200 \mu \mathrm{g}$ & 277 & 242 & 221 & 204 & 193 & 187 & 159 \\
\hline Aclidinium $400 \mu \mathrm{g}$ & 269 & 232 & 220 & 208 & 196 & 189 & 156 \\
\hline
\end{tabular}

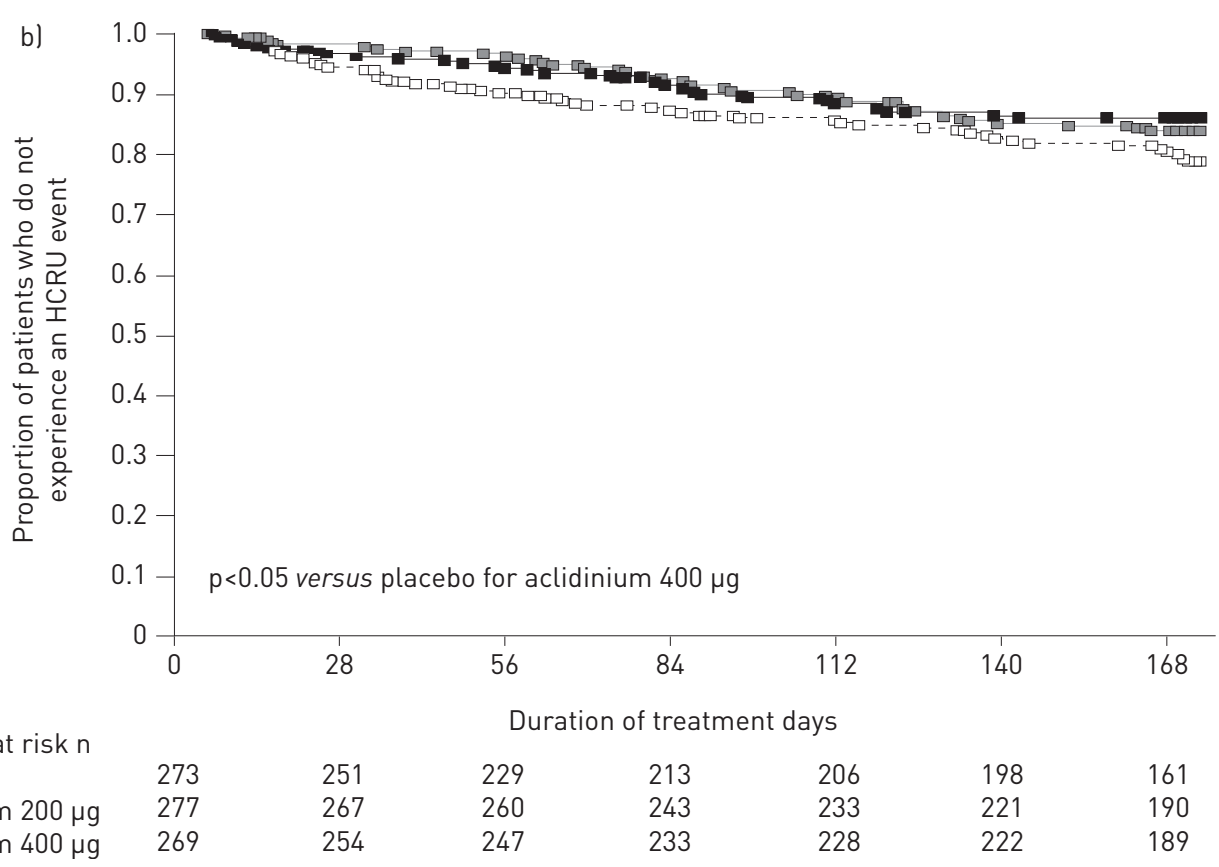

Patients at risk $n$

\begin{tabular}{|c|c|c|c|c|c|c|c|}
\hline Placebo & 273 & 251 & 229 & 213 & 206 & 198 & 161 \\
\hline Aclidinium $200 \mu \mathrm{g}$ & 277 & 267 & 260 & 243 & 233 & 221 & 190 \\
\hline Aclidinium $400 \mu \mathrm{g}$ & 269 & 254 & 247 & 233 & 228 & 222 & 189 \\
\hline
\end{tabular}

FIGURE 4 Kaplan-Meier curves for time to first a) EXAcerbations of Chronic pulmonary disease Tool (EXACT)identified event, and b) healthcare resource utilisation (HCRU) event. Aclidinium was taken twice daily; intent-to-treat population.

impact of unreported events in the context of a clinical trial. The key findings were: 1) there were more than twice as many unreported EXACT events as HCRU events, and agreement between HCRU and the EXACT was poor; 2) patients with unreported (and therefore untreated) EXACT events appeared to recover less well by 28 days post-event than those with reported (and treated) EXACT events, even though these patients had a lower symptom level pre-exacerbation; 3) unreported (untreated) EXACT events had the same medium-term health consequences as reported (treated) HCRU events; and 4) maintenance bronchodilator treatment reduced the rate of both EXACT-identified and HCRU events. Our results confirm and significantly extend previous findings from cohort studies $[3,9-11,18]$ and a recent study using the EXACT [12].

The very large difference in the annualised rate of HCRU and EXACT-identified events observed both here and previously [12] is not fully understood. The EXACT is a standardised symptom assessment instrument 

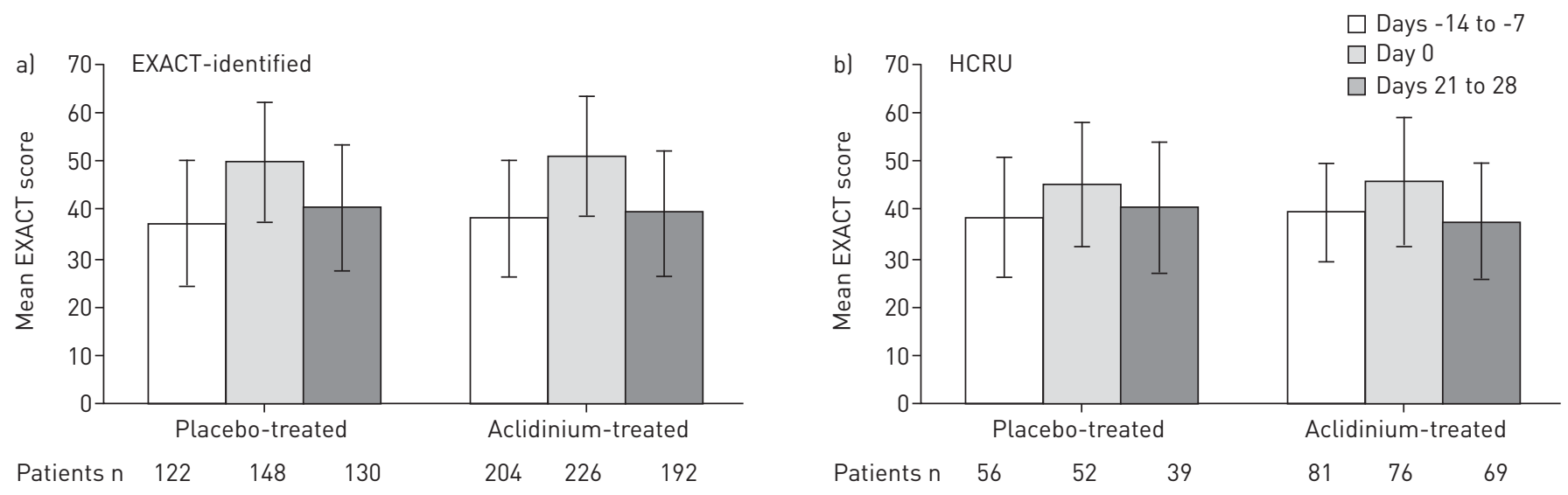

FIGURE 5 Mean EXAcerbations of Chronic pulmonary disease Tool (EXACT) scores in placebo- and aclidinium-treated (200 and $400 \mu \mathrm{g}$ twice daily) patients at days -14 to -7, day 0 and days 21 to 28 relative to onset (day 0) of a) an EXACT-identified event, and b) a healthcare resource utilisation (HCRU) event. Intentto-treat population. Data are presented as mean \pm SD.

designed to measure every patient in the same way. By contrast, reporting an ECOPD has a behavioural component that may be influenced by attitude to illness, expectation of recovery, social circumstances and local healthcare organisations, among other factors. Support for the potential influence of regional variations comes from differences observed between countries in the rates of HCRU and EXACT-identified events; these variations are predominantly due to differences in the rates of HCRU events rather than EXACT events. Our data also suggest that the degree of acute deterioration is not a factor that prompts patients to seek medical advice, as EXACT scores worsened by a similar amount in EXACT-reported and EXACT-unreported events and are consistent with those reported previously $[12,19]$. By contrast, absolute symptom severity may be a factor, as patients with EXACT-reported events had a worse EXACT score when stable and at event onset compared with patients who had unreported EXACT events, an observation also reported previously [12]. Furthermore, patients with HCRU events had lower predicted FEV1 at baseline than patients with EXACT-identified events. Therefore, it appears that patients with milder COPD may be less likely to seek medical attention for an ECOPD, although inter-group differences were small. The speed of deterioration appears to be faster with unreported versus reported events. This has also been observed previously [12]; however, the apparent differences may be an artefact as the first day of an exacerbation was determined differently for the two types of event.

Agreement between HCRU and the EXACT was low. This is consistent with data from a randomised controlled trial that reported limited agreement between exacerbation events identified based on medical intervention and those identified by diary cards [20]. In our study, the lack of agreement between methods was independent of the severity of HCRU events and was largely due to the higher number of patients with unreported EXACT events, but was also due to the fact that more than 50\% of HCRU events did not achieve a change in EXACT score that met the criteria for an EXACT event. The reason for this is not fully clear. In some cases, this was because patients did not complete the diary, particularly those who were

TABLE 3 Changes from baseline at week 24 in SGRQ total score and trough FEV 1 in patients who experienced an EXACTunreported event, an HCRU event or no exacerbation event in the first 12 weeks

\begin{tabular}{|c|c|c|c|c|c|}
\hline & \multirow[t]{2}{*}{ No exacerbation event } & \multicolumn{2}{|c|}{ EXACT-unreported event } & \multicolumn{2}{|c|}{ HCRU event } \\
\hline & & Data & p-value $\#$ & Data & p-value ${ }^{\#}$ \\
\hline Patients $\mathrm{n}$ & 576 & 159 & & 84 & \\
\hline Change from baseline in SGRQ total score & $-6.64 \pm 0.57^{+}$ & $-3.01 \pm 1.06$ & 0.002 & $-3.43 \pm 1.44$ & 0.036 \\
\hline SGRQ responders at week $24^{\pi} \%$ & $54.7^{+}$ & 43.4 & $0.009^{\S}$ & 44.1 & $0.056^{\S}$ \\
\hline Change from baseline in trough FEV1 $\mathrm{mL}$ & $12 \pm 11$ & $-22 \pm 21$ & 0.139 & $-10 \pm 29$ & 0.475 \\
\hline
\end{tabular}

Data are from the intent-to-treat population with all treatment arms combined and are presented as least Square mean $\pm \mathrm{SE}$, unless otherwise stated. SGRQ: St George's Respiratory Questionnaire; FEV1: forced expiratory volume in 1 s; EXACT: EXAcerbations of Chronic pulmonary disease Tool; HCRU: healthcare resource utilisation. " : versus no event; ": patients achieving a clinically significant improvement in SGRQ total score $1 \geqslant 4$ points); ${ }^{+}: \mathrm{n}=572 ;^{\S}$ : odds ratio $\mathrm{p}$-value. 
TABLE 4 Treatment differences versus placebo at week 24 in SGRQ total score, and percentage of SGRQ responders, in patients who experienced an EXACT-unreported event, an HCRU event or no exacerbation event in the first 12 weeks

\begin{tabular}{|c|c|c|c|c|c|c|c|c|}
\hline & \multicolumn{2}{|c|}{ Placebo } & \multicolumn{3}{|c|}{ Aclidinium $200 \mu \mathrm{g}$} & \multicolumn{3}{|c|}{ Aclidinium $400 \mu \mathrm{g}$} \\
\hline \multicolumn{9}{|l|}{$\begin{array}{l}\text { SGRQ total score (differences } \\
\text { versus placebo) }\end{array}$} \\
\hline EXACT-unreported event & 61 & & 52 & $-0.94(-5.84-3.95)$ & 0.704 & 46 & $-3.48(-8.52-1.55)$ & 0.174 \\
\hline HCRU event & 35 & & 23 & $-3.08(-10.81-4.66)$ & 0.431 & 26 & $-3.61(-11.17-3.95)$ & 0.345 \\
\hline EXACT-unreported event & & 36.1 & & 46.2 & $0.358^{+}$ & & 50.0 & $0.169^{+}$ \\
\hline HCRU event & & 37.1 & & 47.8 & $0.327^{+}$ & & 50.0 & $0.436^{+}$ \\
\hline No exacerbation & & 43.4 & & 59.5 & $0.002^{+}$ & & 59.9 & $0.003^{+}$ \\
\hline
\end{tabular}

Data are from the intent-to-treat population and are presented as least squares mean difference versus placebo ( $95 \%$ confidence interval), unless otherwise stated. Aclidinium was taken twice daily. SGRQ: St George's Respiratory Questionnaire; EXACT: EXAcerbations of Chronic pulmonary disease Tool; HCRU: healthcare resource utilisation. " : versus placebo; ${ }^{\uparrow}$ : patients achieving a clinically significant improvement in SGRQ total score $\left(\geqslant 4\right.$ points); ${ }^{+}$: odds ratio $p$-value.

hospitalised. It is possible that other factors, not reflected in the diary card, may influence a patient's decision to change their treatment or seek medical advice, or some patients may consider worsening of one or two specific symptoms very highly, and much more highly than the weight accorded by the EXACT. The EXACT electronic diary required daily entry so that missing data could be detected. The low degree of concordance was not necessarily a failure of the EXACT. As in all studies that record HCRU events, the clinician makes a diagnosis based on the patient's history supplemented by physical examination. To our knowledge, there has never been a formal study of inter-rater reliability of a clinical ECOPD diagnosis and no biomarker is currently available to confirm an exacerbation.

This study is the first to show that treatment reduced both reported and unreported exacerbations. It does not alter the role of HCRU exacerbations as an outcome in COPD trials, but it does show that unreported events appear to have important short- to medium-term consequences in terms of symptoms and health status. There was no difference between reported and unreported events in terms of effect on FEV1, but this may have been because FEV1 had recovered 3 months after the event and the relationship between reduction in exacerbations and improvement in FEV1 is weak [21]. Further studies are needed to examine the impact of unreported EXACT events on outcomes such as FEV1 decline and exercise capacity and to test whether unreported exacerbations are predictors of future events. If future studies showed that EXACT events have important clinical consequences, there would need to be agreement as to which measure of exacerbations should be used.

The NNT for prevention of one exacerbation event per patient per year was lower for all EXACT-identified events than for HCRU events (i.e. treatment was more effective). This treatment effect may be important because more than three-quarters of EXACT events were unreported and untreated. Furthermore, those events had a similar impact on health status to those that were reported and treated. Finally, it may take longer to recover from an untreated event, as reported here and previously [11].

A limitation of this study is that annualised event rates were based on 6 months' study treatment; however, a large meta-analysis showed that the proportion of patients who experienced an exacerbation in randomised trials of tiotropium of $<1$ year's duration was similar to that in studies of $\geqslant 1$ year [22]. Furthermore, as one of the key aims of these analyses was to compare exacerbations assessed using two different measures within the same study, it may be assumed that the same limitations will apply to events assessed by both the EXACT and HCRU. The ATTAIN study was not powered to detect differences in exacerbation rate and patients recruited to the study were not required to have a history of ECOPDs ( $35 \%$ had $\geqslant 1$ exacerbation in the year prior to the study [13]). Despite this, treatment with aclidinium twice daily reduced exacerbation events, irrespective of assessment method, suggesting that the observed effects on exacerbations are robust. A further limitation is that the analyses comparing treated and untreated exacerbation events were exploratory and performed in the minority of patients who had an exacerbation. These analyses are the first of their kind and, although they need replication, they suggest that use of the EXACT provides new and important insights, and strongly support the use of this instrument in other studies. 
In conclusion, unreported COPD exacerbation events, identified using the EXACT, were more common than exacerbations identified using HCRU. Unreported events had a similar impact on health status 3-6 months post-event as reported events. Twice-daily treatment with aclidinium (200 $\mu \mathrm{g}$ or $400 \mu \mathrm{g}$ ) reduced EXACT events and HCRU events by a similar proportion.

\section{Acknowledgements}

The authors thank all the ATTAIN study investigators and PAREXEL International Limited, Uxbridge, UK. We also thank Deborah McGregor from Complete Medical Communications, Macclesfield, UK, who provided medical writing support funded by Almirall S.A., Barcelona, Spain.

\section{References}

1 Vestbo J, Hurd SS, Agustí AG, et al. Global strategy for the diagnosis, management, and prevention of chronic obstructive pulmonary disease: GOLD executive summary. Am J Respir Crit Care Med 2013; 187: 347-365.

2 Donaldson GC, Seemungal TA, Bhowmik A, et al. Relationship between exacerbation frequency and lung function decline in chronic obstructive pulmonary disease. Thorax 2002; 57: 847-852.

3 Seemungal TA, Hurst JR, Wedzicha JA. Exacerbation rate, health status and mortality in COPD - a review of potential interventions. Int J Chron Obstruct Pulmon Dis 2009; 4: 203-223.

4 Toy EL, Gallagher KF, Stanley EL, et al. The economic impact of exacerbations of chronic obstructive pulmonary disease and exacerbation definition: a review. COPD 2010; 7: 214-228.

5 Cazzola M, MacNee W, Martinez FJ, et al. Outcomes for COPD pharmacological trials: from lung function to biomarkers. Eur Respir J 2008; 31: 416-469.

6 Burge S, Wedzicha JA. COPD exacerbations: definitions and classifications. Eur Respir J 2003; 21: Suppl. 41, 46s-53s.

7 Rodriguez-Roisin R. Toward a consensus definition for COPD exacerbations. Chest 2000; 117: Suppl. 2, 398S-401S.

8 Leidy NK, Wilcox TK, Jones PW, et al. Development of the EXAcerbations of Chronic obstructive pulmonary disease Tool (EXACT): a patient-reported outcome (PRO) measure. Value Health 2010; 13: 965-975.

9 Langsetmo L, Platt RW, Ernst P, et al. Underreporting exacerbation of chronic obstructive pulmonary disease in a longitudinal cohort. Am J Respir Crit Care Med 2008; 177: 396-401.

10 Seemungal TA, Donaldson GC, Paul EA, et al. Effect of exacerbation on quality of life in patients with chronic obstructive pulmonary disease. Am J Respir Crit Care Med 1998; 157: 1418-1422.

11 Wilkinson TM, Donaldson GC, Hurst JR, et al. Early therapy improves outcomes of exacerbations of chronic obstructive pulmonary disease. Am J Respir Crit Care Med 2004; 169: 1298-1303.

12 Leidy NK, Murray LT, Jones P, et al. Performance of the EXAcerbations of Chronic pulmonary disease Tool patient-reported outcome measure in three clinical trials of chronic obstructive pulmonary disease. Ann Am Thorac Soc 2014; 11: 316-325.

13 Jones PW, Singh D, Bateman ED, et al. Efficacy and safety of twice-daily aclidinium bromide in COPD patients: the ATTAIN study. Eur Respir J 2012; 40: 830-836.

14 Leidy NK, Wilcox TK, Jones PW, et al. Standardizing measurement of chronic obstructive pulmonary disease exacerbations. Reliability and validity of a patient-reported diary. Am J Respir Crit Care Med 2011; 183: 323-329.

15 Jones PW, Chen WH, Wilcox TK, et al. Characterizing and quantifying the symptomatic features of COPD exacerbations. Chest 2011; 139: 1388-1394.

16 EXACT-PRO Initiative. The Exacerbations of Chronic pulmonary disease Tool (EXACT) User Manual Version 4.0. Bethesda, Evidera, 2013.

17 Spencer S, Jones PW. Time course of recovery of health status following an infective exacerbation of chronic bronchitis. Thorax 2003; 58: 589-593.

$18 \mathrm{Xu} \mathrm{W}$, Collet JP, Shapiro S, et al. Negative impacts of unreported COPD exacerbations on health-related quality of life at 1 year. Eur Respir J 2010; 35: 1022-1030.

19 Halpin DM, Laing-Morton T, Spedding S, et al. A randomised controlled trial of the effect of automated interactive calling combined with a health risk forecast on frequency and severity of exacerbations of COPD assessed clinically and using EXACT PRO. Prim Care Respir J 2011; 20: 324-331.

20 Calverley P, Pauwels R, Löfdahl CG, et al. Relationship between respiratory symptoms and medical treatment in exacerbations of COPD. Eur Respir J 2005; 26: 406-413.

21 Jones PW, Donohue JF, Nedelman J, et al. Correlating changes in lung function with patient outcomes in chronic obstructive pulmonary disease: a pooled analysis. Respir Res 2011; 12: 161.

22 Karner C, Chong J, Poole P. Tiotropium versus placebo for chronic obstructive pulmonary disease. Cochrane Database Syst Rev 2012; 7: CD009285. 